\title{
Research on Variable Inertia Coordination Frequency Regulation Strategy Based on a Wind \& Thermal Power Combined System
}

\author{
Siqing Sheng ${ }^{1}$, Pengwang $\mathrm{Li}^{1,}{ }^{*}$, Hao $\mathrm{Wu}^{1}$, Liwei Zhang ${ }^{1}$, and Maosen $\mathrm{Fan}^{1}$ \\ ${ }^{1}$ School of Electrical \& Electronic Engineering, North China Electric Power University, Baoding 071003, Hebei Province, China
}

\begin{abstract}
With the increasing installed capacity of the wind power, the power system has an obviously low inertia characteristic. It is of great significance to actively promote the virtual inertia frequency regulation technology of wind turbines (WTS) for improving the system frequency quality. The frequency regulation capability and frequency regulation effects of wind \& thermal power units were analysed, and a variable inertia coordination frequency regulation strategy for different wind power penetration conditions was proposed in this paper. At the wind farm level, the dynamic frequency regulation participation coefficient of wind farms was fuzzily determined according to the operation conditions of WTS and the wind power penetration ratio. At the wind turbine level, the calculation method of the equivalent inertia constant of WTS was given based on the effective rotational kinetic energy. And the allowable range of frequency regulation parameters of WTS was determined by considering the incremental model of the system. Results indicated that the proposed coordinated frequency regulation strategy not only provided a reliable inertia support, but also maintained the stability of WTS. The frequency response performance of the highpenetration wind power system was improved.
\end{abstract}

\section{Introduction}

Wind power, as a relatively mature sustainable energy generation mode, has an increasing penetration proportion in the power system ${ }^{[1]}$. The large-scale wind power accesses to the grid greatly weakens the frequency regulation capability of the system ${ }^{[2-3]}$. The frequency regulation ability of wind turbines (WTS) is stipulated in the latest grid guidelines issued at home and abroad to improve the frequency regulation capability of the highly penetrated wind power system ${ }^{[4]}$.

The auxiliary frequency regulation strategy of WTS had been studied by some scholars. At the wind turbine level, the active output of WTS or energy storage systems is changed to respond to the frequency fluctuation [5]. The specific implementation measures include: virtual inertia control ${ }^{[6-8]}$, active power reserve control ${ }^{[8-10]}$, multi-strategy coordination control [11-12] and the virtual synchronous generator technology ${ }^{[13-14]}$, which all effectively improve the system frequency quality. The virtual inertia control of WTS can rapidly increase active output to provide frequency support and have better operational economy ${ }^{[5,15]}$. Therefore, the frequency regulation capability and frequency regulation effects of WTS with virtual inertia control are studied in this paper. The reference [16] proposed a frequency regulation strategy of dynamically adjusting the virtual inertia control's parameters by tracking the frequency state. That strategy made the virtual inertia control of WTS better adapted to the system frequency variation, which greatly utilized the frequency regulation potential of WTS. However, the virtual inertia control's parameters might be designed extremely sensitive to maximize the frequency regulation capability of WTS in previous studies. Based on this, a variable parameter frequency regulation control method was proposed in reference [17] according to the operation status of WTS. This method could avoid the secondary frequency drop and ensured the safe and stable operation of the system. However, these control methods are cumbersome, which are difficult to adapt to engineering application and without considering the stability of wind turbine control itself.

At the wind farm level, previous studies have shown that the inertia response of wind farms is a multi-unit coordinated control problem ${ }^{[18]}$. Regarding this, the paper [19] divided WTS into zones according to wind speed, and then the short-term additional power of the wind farm was dynamically allocated, thus the frequency response performance was improved. The reference [20] further coordinated and optimized the short-term output of the wind and storage joint system by using the fuzzy control method and improved the frequency stability. However, the above literatures are all carried out under a constant wind power penetration, without considering the applicability of frequency regulation control schemes under different wind power penetration conditions.

To sum up, based on the analysis of the frequency regulation effect of wind farms and thermal power units, the variable inertia coordination frequency regulation strategy for different wind power penetration conditions is explored. At the wind farm level, based on the fuzzy logic rules, a frequency regulation strategy of dynamic

\footnotetext{
* Corresponding author: LPW5806@163.com
} 
decision-making frequency regulation participation coefficient of wind farms is proposed according to the operation conditions of wind farms and the wind power penetration of the system. At the wind turbine level, the variable inertia frequency regulation control scheme is proposed considering the relationship between the equivalent inertia constant of WTS and the incremental model of the wind and thermal joint system. The results show that the proposed strategy effectively improves the frequency quality and the stability of the system.

\section{Auxiliary frequency regulation control of wind turbines}

\subsection{Analysis of the frequency regulation ability for the wind and thermal power units}

It is necessary to analyse the frequency regulation capability of the wind and thermal power units to study the frequency regulation control of the wind and thermal power combined system. At present, the doubly-fed induction generator (DFIG) is the main model of wind farms. Therefore, this paper takes the DFIG operating in the MPPT area ${ }^{[2]}$ as the research object, and its typical values can be found in reference [6]. The active output curves of wind and thermal power units when the system frequency drops are shown in Fig.1.

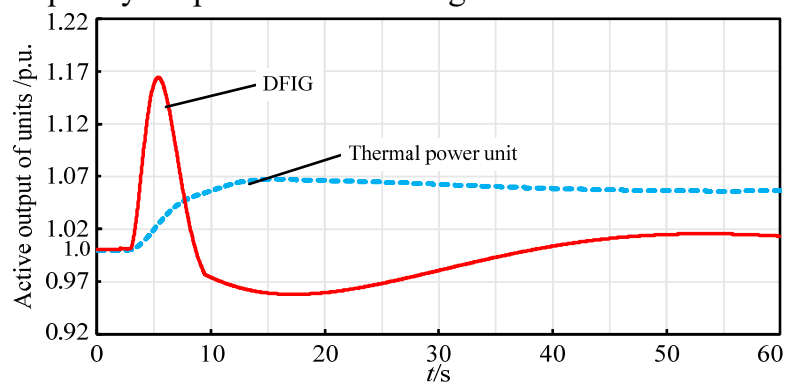

Fig. 1 Active output curves of wind and thermal power units

As shown in Fig.1, DFIGs can quickly provide larger active support at the initial time of the power disturbance, but the duration of active support is relatively short, which is only about $3.8 \mathrm{~s}$ in this case. And additional active power will be absorbed from system in the frequency recovery stage, which will lead to serious secondary frequency drop when the wind power penetration ratio is high enough. However, thermal power units have larger inertia time constant. Although they cannot quickly damp the frequency variation, they can maintain stable active output in a longer period.

In summary, the fast frequency regulation characteristic of wind farms and the frequency regulation sustainability of thermal power units should be fully utilized. Therefore, the inertia and damping of the system should be weakened as far as possible to avoid serious secondary frequency drop in the frequency recovery stage and to accelerate the frequency recovery.

\subsection{Frequency regulation model of the high- penetration wind power system}

Based on the above analysis, a coordinated frequency regulation model is proposed as shown in Fig.2. In the Fig.2, $\Delta P_{\mathrm{m}}$ is the active increment of thermal power units, $\Delta P_{\mathrm{w}}$ is the active increment of DFIGs, $\Delta P_{\omega}$ is the increment of wind power capture, $P_{\mathrm{L}}$ is the load power; $K_{\mathrm{m}}$ and $K_{\mathrm{w}}$ are the frequency regulation participation coefficient of thermal power units and wind farms respectively; $\omega_{\mathrm{w} 0}$ is the initial speed of DFIGs, $\Delta \omega_{\mathrm{w}}$ is the speed increment, $k_{\text {opt }}$ is the coefficient of the MPPT curve $^{[2]} ; K_{\mathrm{df}}$ and $K_{\mathrm{pf}}$ are the differential coefficient and proportional coefficient of auxiliary frequency regulation.

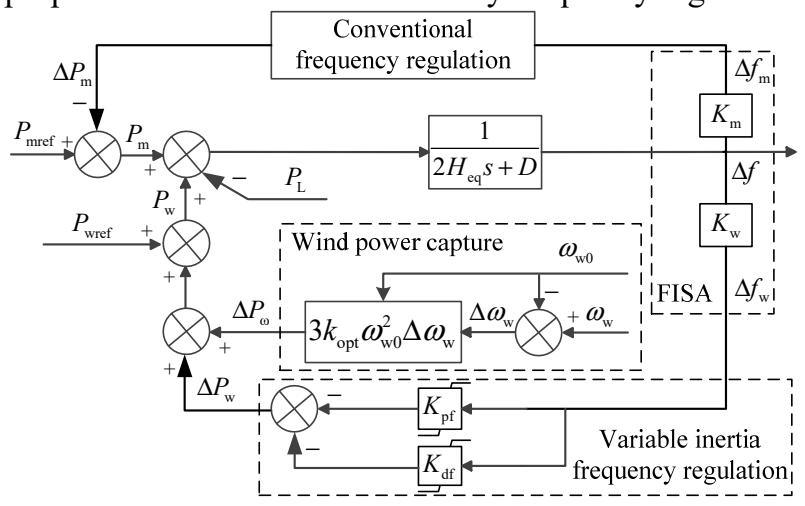

Fig. 2 The frequency regulation model of the system

In the Fig.2, the equivalent rotor motion equation of the system can be obtained as follows:

$$
2 H_{\mathrm{eq}} \frac{d \Delta f}{d \mathrm{t}}=P_{\mathrm{m}}+P_{\mathrm{w}}-P_{\mathrm{L}}-D \Delta f
$$

Where, $\Delta f$ is the frequency deviation, $H_{\mathrm{eq}}$ is the equivalent inertial constant, $D$ is the system damping.

When the $\Delta P_{\omega}$ is neglected, the dynamic frequency response equation of the system is as follows:

$$
\begin{aligned}
\left(2 H_{\mathrm{eq}}+K_{\mathrm{w}} K_{\mathrm{df}}\right) \frac{\mathrm{d} \Delta f}{\mathrm{dt}} & =P_{\mathrm{m}}-P_{\mathrm{L}}+P_{\mathrm{wref}}+\Delta P_{\omega} \\
& -\left(D+K_{\mathrm{w}} K_{\mathrm{pf}}\right) \Delta f
\end{aligned}
$$

The equation (2) shows that the equivalent inertia of the system increases $K_{\mathrm{w}} K_{\mathrm{df}}$ and the system damping increases $K_{\mathrm{w}} K_{\mathrm{pf}}$ when the frequency regulation parameters $K_{\mathrm{df}}$ and $K_{\mathrm{pf}}$ are both positive. However, the further analysis shows that the increase of the equivalent inertia and damping of the system will reduce the frequency fluctuation when the system frequency deteriorates. And it is necessary to reduce the equivalent inertia and damping to accelerate the frequency recovery process when the system frequency restores.

Therefore, it is necessary to adjust the parameters $K_{\mathrm{df}}$ and $K_{\mathrm{pf}}$ with real time according to the frequency fluctuation state of the system for improving the system frequency characteristic.

\section{Variable inertia coordination frequen- cy regulation strategy of the wind and thermal power combined system}

\subsection{The effective kinetic energy of DFIGs}

Combining with Figs.1 and 2, the auxiliary inertia frequency regulation of DFIGs is realized by adjusting 
the rotor speed. The rotor speed has a great influence on the inertia of DFIGs and affects the captured wind power. The change of the effective kinetic energy includes two parts. One is the change of the actual kinetic energy $\Delta E_{\mathrm{k}}$ caused by the change of the speed, and the other is the change of the captured wind power $\Delta E_{\mathrm{w}}$. Therefore, the effective kinetic energy $\Delta E_{\mathrm{keq}}$ is:

$$
\Delta E_{\mathrm{keq}}=\Delta E_{\mathrm{k}}+\Delta E_{\mathrm{w}}
$$

The variation of the $\Delta E_{\mathrm{keq}}$ is constrained by the rotor speed, and the relation could be explained as below:

$$
\left\{\begin{array}{l}
\Delta E_{\mathrm{k}}=\frac{1}{2} J_{\mathrm{w}}\left(\omega_{\mathrm{w} 0}^{2}-\omega_{\mathrm{wmin}}^{2}\right) \\
\Delta E_{\mathrm{w}}=\int_{t_{0}}^{t_{0}+\Delta t}\left(P_{\mathrm{w}}(t)-P_{\mathrm{w} 0}\right) \mathrm{d} t
\end{array}\right.
$$

Where, $J_{\mathrm{w}}$ is the rotor inertia; $t_{0}$ is the start time for frequency regulation, $\Delta t$ is the frequency regulation duration; $P_{\mathrm{w}}(t)$ is the captured power with real time, $P_{\mathrm{w} 0}$ is the initial captured power of DFIGs.

Under certain conditions, the $\Delta E_{\mathrm{keq}}$ can be expressed by the equivalent inertia constant $H_{\mathrm{wv}}$, and the expression of the $H_{\mathrm{wv}}$ as shown in equation (5).

$$
\begin{aligned}
H_{\mathrm{wv}}= & \frac{1}{P_{\text {wrated }}}\left\{\frac{1}{2} J_{\mathrm{w}}\left(\omega_{\mathrm{w} 0}^{2}-0.49\right)-\int_{t_{0}}^{t_{0}+\Delta t}\left[\frac{1}{2} \rho S C_{\text {popt }} V_{\mathrm{w}}^{3}+0.0068 \frac{\omega_{\mathrm{w}} R}{V_{\mathrm{w}}}\right.\right. \\
& \left.\left.-\frac{1}{2} \rho S V_{\mathrm{w}}^{3}\left(0.5176\left(116\left(\frac{V_{\mathrm{w}}}{\omega_{\mathrm{w}} R}-0.035\right)-5\right) \mathrm{e}^{21\left(0.035-V_{\mathrm{w}} / \omega_{\mathrm{w}} R\right)}\right)\right] \mathrm{d} t\right\}
\end{aligned}
$$

Suppose the rotor speed of DFIGs changes from the $\omega_{\mathrm{w} 0}$ to 0.7 p.u., the $H_{\mathrm{wv}}$ determined by equation (5) is the largest, defined as the maximum inertia constant $H_{\mathrm{wm}}$, which is related to the $\omega_{\mathrm{w} 0}$ and the wind speed $V_{\mathrm{w}}$. And the relation between the $H_{\mathrm{wm}}$ and $\omega_{\mathrm{w}}$ as shown in Fig.3.

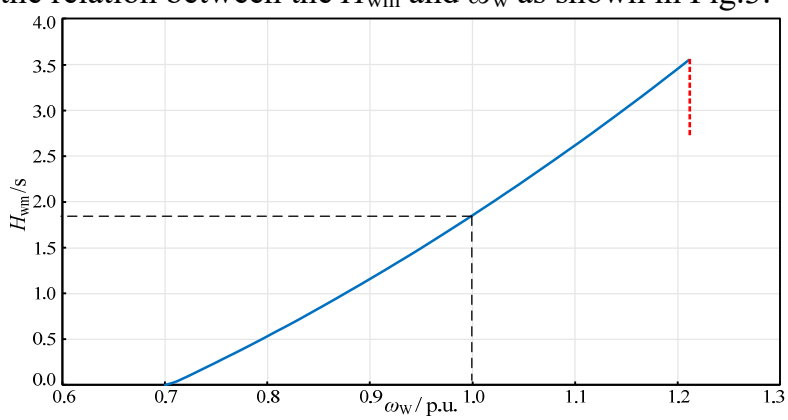

Fig. 3 The relational curve between the $H_{\mathrm{wm}}$ and $\omega_{\mathrm{w}}$

In summary, the $H_{\mathrm{wm}}$ of DFIGs is different under different operating conditions. The greater the initial speed before the frequency regulation implementing is, the greater the virtual inertia will be. Therefore, it is necessary to set the maximum value of the $K_{\mathrm{df}}$ according to different operating conditions to avoid excessive frequency regulation of DFIGs and take full advantage of the frequency regulation potential of DFIGs.

\subsection{Variable inertia coordination frequency reg- ulation strategy based on the fuzzy logic control}

\subsubsection{Frequency regulation strategy of wind farms}

As shown in Fig.2, the frequency regulation participation coefficient $K_{\mathrm{w}}$ is determined by using the fuzzy logic controller FISA. And the $K_{\mathrm{m}}+K_{\mathrm{w}}=1$ is taken as a constraint condition to obtain the reasonable proportion of active output for wind farms and thermal power units. For the FISA, its inputs are the wind power penetration $\alpha_{\mathrm{w}}(0.02 \sim 1)$ and the local average wind speed $V_{\mathrm{w}}$ $(6 \sim 10 \mathrm{~m} / \mathrm{s})$; its output is the frequency regulation participation coefficient of wind farms $K_{\mathrm{w}}(0 \sim 1)$.

The FISA not only makes full use of the frequency regulation potential of wind farms, but also avoids excessive dependence on wind farms, thus the frequency stability of the system is guaranteed. The inference principles of FISA are: when the wind speed $V_{\mathrm{w}}$ is very small or the wind power penetration $\alpha_{\mathrm{w}}$ is very large, the output $K_{\mathrm{w}}$ should be as small as possible; when the wind speed $V_{\mathrm{w}}$ is large and the wind power penetration $\alpha_{\mathrm{w}}$ is small, the output $K_{\mathrm{w}}$ is larger; when the wind speed $V_{\mathrm{w}}$ is large and the wind power penetration $\alpha_{\mathrm{w}}$ is large, the output $K_{\mathrm{w}}$ is very large. In summary, the inference result is obtained as shown in Fig.4.

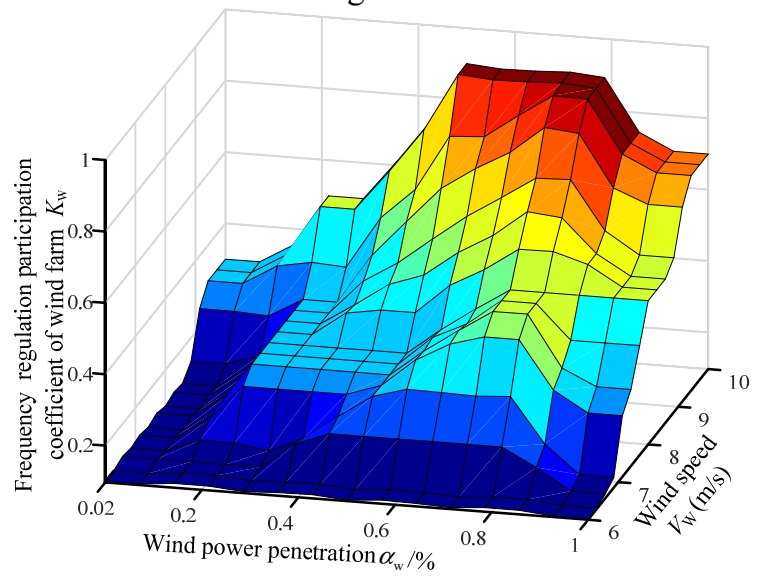

Fig. 4 The inference result of FISA

\subsubsection{Frequency regulation strategy at the wind turbine level}

An incremental model of the wind and thermal power joint system as shown in Fig.5 is established considering that the real-time change of the frequency regulation parameters of DFIGs mays affect the overall stability of the system, which determines the reasonable range of the parameters. The values of these parameters in Fig. 5 are shown in Table A in the Appendix.

Under the rated wind speed $\left(V_{\mathrm{w}}=9.5 \mathrm{~m} / \mathrm{s}\right)$, the $K_{\mathrm{df}}$ and $K_{\text {pf }}$ are set to be 1 , the eigenvalue analysis in frequency domain is carried out with the changing values of the

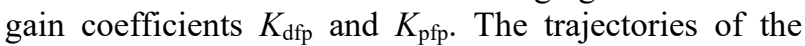
dominant eigenvalues are drawn as shown in Fig.6.

The Fig. 6 shows that the $\lambda_{1}$ is positive when the $K_{\mathrm{pfp}}$ is small. Formula (2) shows that the size of the $K_{\text {pfp }}$ is related to the system damping. If the $K_{\mathrm{pfp}}$ is too small, the system damping will be reduced and the system stability will be poor. When the $K_{\mathrm{pfp}}$ increases, the $\lambda_{1}$ will move to the left half plane and the system stability will be improved. When the value of the $K_{\text {pfp }}$ is large, the $\lambda_{2}$ decreases until it moves to the right half plane, which leads to the instability of DFIG control itself. Similarly, the characteristics of the $\lambda_{3}$ and $\lambda_{4}$ also limit the range of 
the $K_{\mathrm{dfp}}$. It can be concluded that: (1) the range of the $K_{\mathrm{pfp}}$ is (-7 19); (2) the range of the $K_{\text {dfp }}$ is (-9 11). Therefore, the stability of the system can be ensured when taking the subset of the above ranges as the range of the variable inertia frequency regulation parameters.

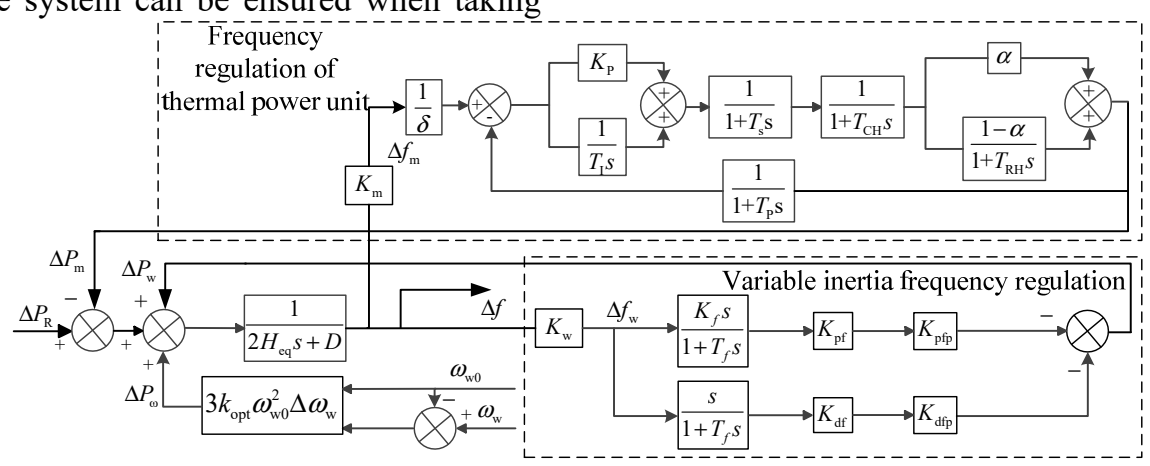

Fig. 5 The incremental model of the wind and thermal joint system

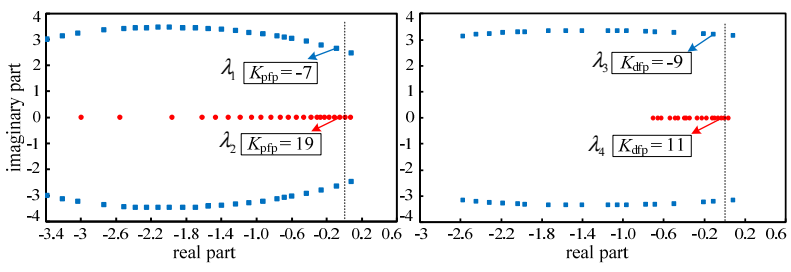

Fig. 6 The change of eigenvalues along with the $K_{\mathrm{pfp}}$ and $K_{\mathrm{dfp}}$

To sum up, this paper presents a variable inertia coordination frequency regulation strategy at the wind turbine level. The control structure is shown in Fig.7. In the Fig.7, the variable inertia frequency regulation parameters are dynamically adjusted by using the fuzzy logic controller FISB. For the FISB, the system frequency deviation $\Delta f(-0.005 \sim 0$ p.u. $)$ and frequency change rate $\mathrm{d} f / \mathrm{d} t\left(-0.012 \sim 0.012\right.$ p.u./s) ${ }^{[20]}$ are used as inputs to effectively distinguish the system frequency state, and the outputs are the $K_{\mathrm{df}}(0 \sim 1)$ and $K_{\mathrm{pf}}(-6 \sim 6)$.

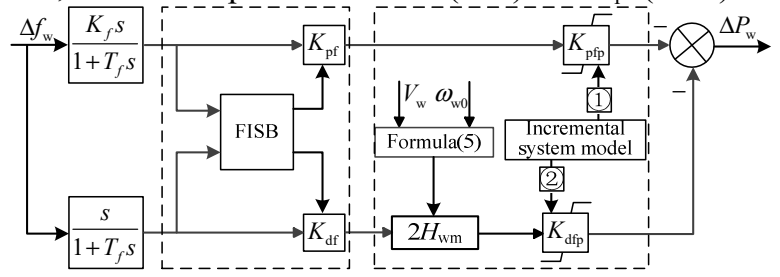

Fig. 7 The frequency regulation control structure at DFIG level

The FISB adjusts the active output of DFIGs with real time according to the frequency variation to compensate the equivalent inertia and damping of the system. The inference principles of FISB are as follows: when the system frequency deviation $\Delta f$ is small and the absolute value of the frequency change rate $\mathrm{d} f / \mathrm{d} t$ is small, the output $K_{\mathrm{df}}$ should be as small as possible, the $K_{\mathrm{pf}}$ is negative and the absolute value should be as large as possible; when the $\Delta f$ is large and the $\mathrm{d} f / \mathrm{d} t$ is positive, the output $K_{\mathrm{df}}$ should be as small as possible, and the $K_{\mathrm{pf}}$ is negative and the absolute value should be as large as possible; when the $\Delta f$ of the system is large and the $\mathrm{d} f / \mathrm{d} t$ of the system is negative, the output $K_{\mathrm{df}}$ should be as large as possible, the $K_{\mathrm{pf}}$ should be positive and the absolute value should be as large as possible, so as to weaken the system frequency fluctuation as much as possible. According to the principles, the corresponding result is obtained as shown in Fig.8.

\section{Analysis of simulation results}

A simulation system is established as shown in Fig.9 by using MATLAB/Simulink to verify the effectiveness of the variable inertia coordination frequency regulation strategy proposed in this paper.

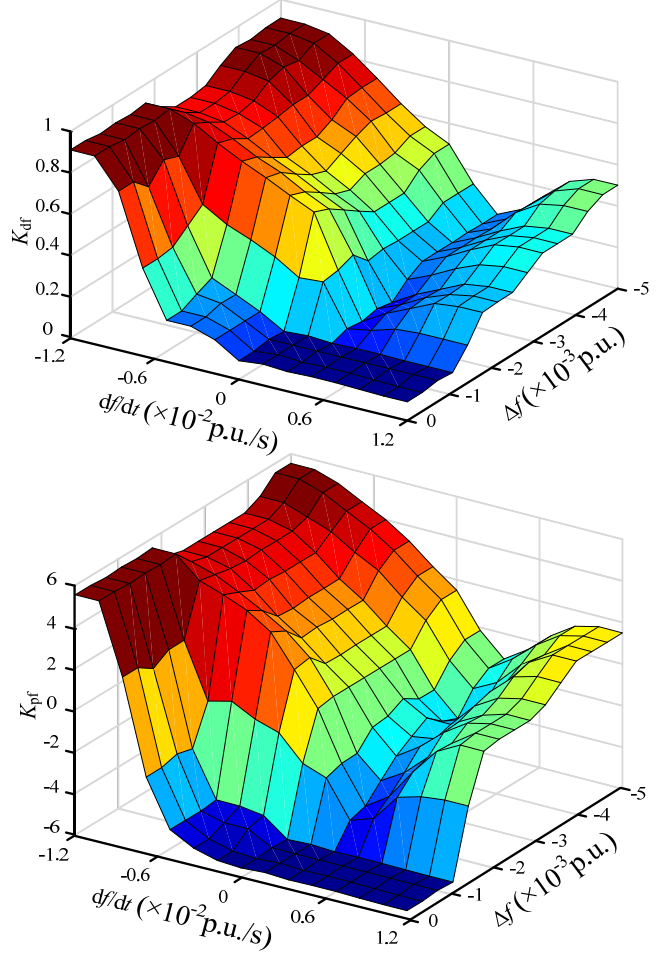

Fig. 8 The inference result of FISB

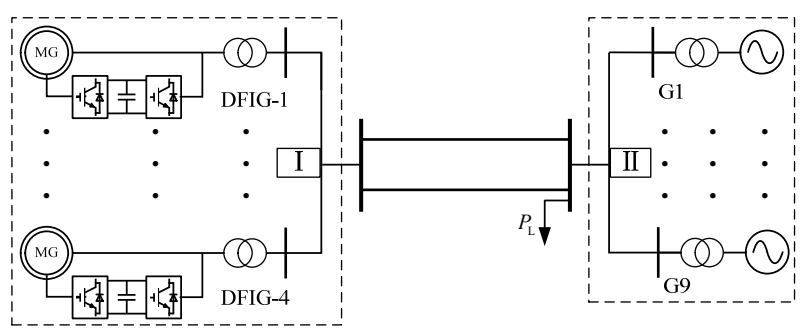

Fig. 9 The structure diagram of the simulation system 
Area I is a wind farm with an installed capacity of $400 \mathrm{MW}$. It consists of four sets of single-machine equivalent models, each of which consists of 40 DFIGs. Area II consists of 9 thermal power units, rated power of single unit is $300 \mathrm{MW}$. The frequency regulation parameters of these units are selected according to Table A in the Appendix. The rated load power $P_{\mathrm{L}}$ is $1600 \mathrm{MW}$.

\subsection{The frequency regulation performance of the system under same wind power penetration rate}

Four groups of DFIGs in Area I are operated in four different wind speed classes (S1 S4), which are divided from high to low according to the wind speed $(6 \sim 10 \mathrm{~m} / \mathrm{s})$ and the principle of $1 \mathrm{~m} / \mathrm{s}$ interval. The total output of DFIGs at each wind speed class is $100 \mathrm{MW}$, and the $\alpha_{\mathrm{w}}$ is $20 \%$. When $\mathrm{t}=2 \mathrm{~s}, 1 \%$ power disturbance occurs in the system. The results are shown in Fig. 10.

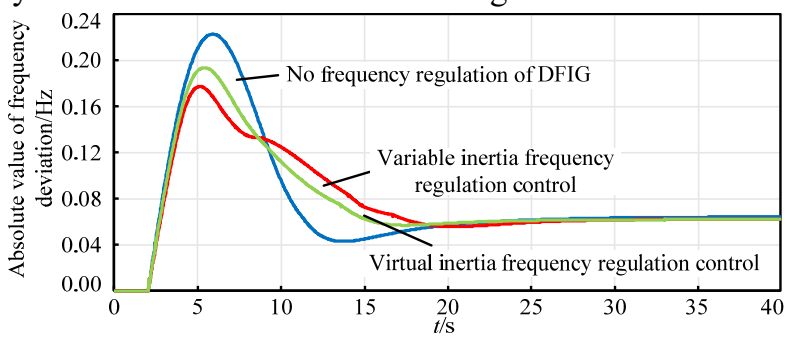

Fig. 10 Changes of the system frequency

As shown in the Fig.10, the maximum frequency deviation of the system decreases when wind farms participate in frequency regulation. And compared with the virtual inertia control technology, the proposed coordination frequency regulation strategy makes the frequency regulation effect of DFIGs is better.

The specific changes of the active output and the speed variation of DFIGs in each wind speed class are shown in Figs. 11 and 12.

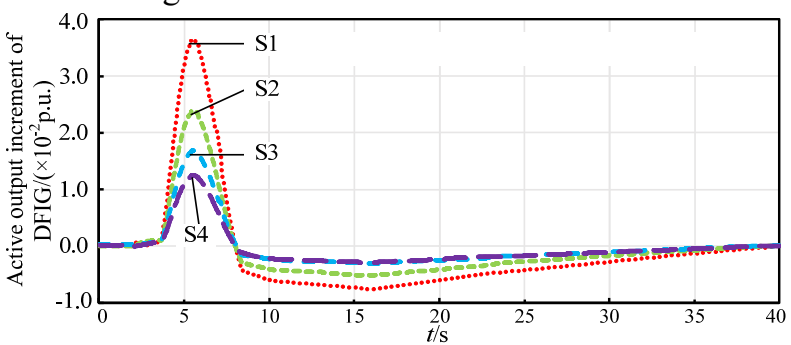

Fig. 11 Active output of DFIGs in different wind speed classes

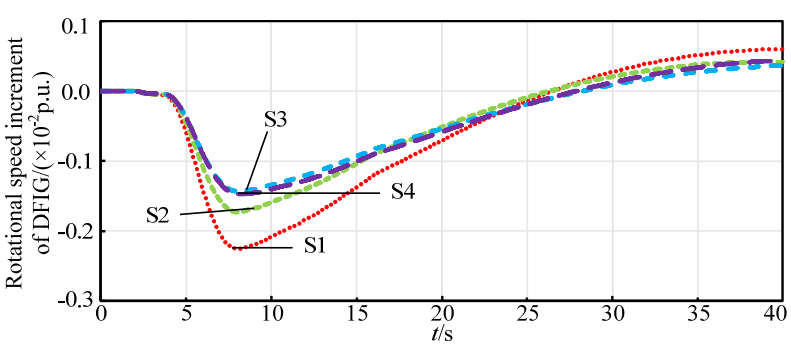

Fig. 12 Rotor speed of DFIGs in different wind speed classes

It can be seen that DFIGs with different wind speeds all can provide active support during frequency fluctuation from the Figs.11 and 12. With the increase of the wind speed class, the short-term active output of DFIGs increases gradually, up to 0.037 p.u., and the duration of active support also increases gradually, up to $5.5 \mathrm{~s}$. That is, the kinetic energy released by DFIGs in a short time increases gradually, which leads to the increase of the speed variation of DFIGs, up to $0.0024 \mathrm{p} . \mathrm{u}$.

The analysis of Figs. 10 and 12 shows that DFIGs enter the speed recovery stage in turn, because of the different maximum inertia of DFIGs with different wind speed classes, which weakens the intensity of the secondary frequency drop to a certain extent.

The simulation results of the first 40s of the FISB are shown in Fig.13. It can be concluded that the rapid increase of the $K_{\mathrm{pf}}$ and $K_{\mathrm{df}}$ can effectively damp the system frequency deterioration and accelerate the system frequency recovery during the frequency declines.

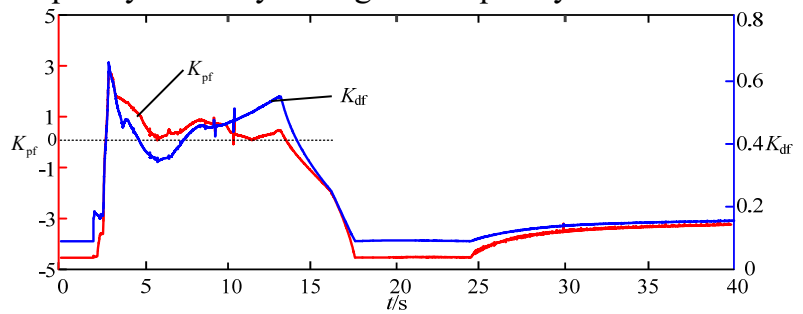

Fig. 13 Output of the FISB

\subsection{The frequency regulation effect under different wind power penetration rates}

Suppose DFIGs in Area I have same wind speed ( $V_{\mathrm{w}}$ $=10 \mathrm{~m} / \mathrm{s})$, the output of the wind farm is constantly changed. The same power disturbance occurs when $\mathrm{t}=2 \mathrm{~s}$ to check the frequency regulation effect of the system and verify the robustness of the proposed frequency regulation strategy. The results are shown in Fig. 14.

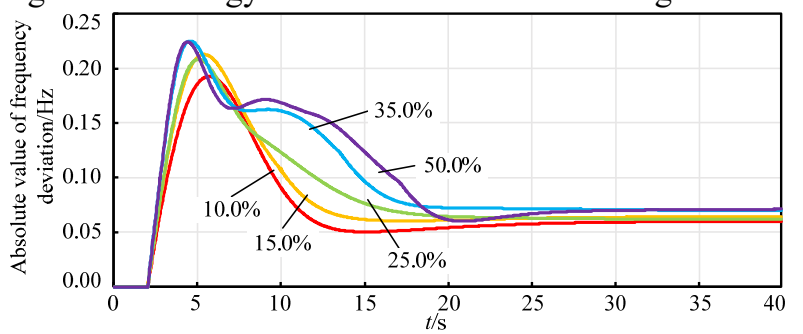

Fig. 14 Frequency curves of the system

The Fig.14 shows that the system frequency can be maintained stable under different conditions, and the system frequency deviation becomes larger and larger with the increase of the wind power penetration $\alpha_{\mathrm{w}}$. That is because the equivalent inertia of the system decreases gradually and the frequency change rate of the system accelerates gradually. At the same time, the serious secondary frequency drop phenomenon is caused because the wind farm undertakes more and more frequency regulation pressure according to the result of FISA's fuzzy inference (see Fig.4). The short-term active output and the rotor speed of DFIGs under different wind power penetration conditions are shown in Table 1. 
Table 1. Simulation results with different wind power penetration conditions

\begin{tabular}{|c|c|c|c|c|}
\hline $\begin{array}{c}\boldsymbol{\alpha}_{\mathbf{w}} \\
\mathbf{\%} \%\end{array}$ & $\boldsymbol{K}_{\mathbf{w}} / \mathbf{p . u .}$ & $\begin{array}{c}\text { Active } \\
\text { Output } \\
\text { Increment } \\
\text { of DFIGs } \\
\text { /p.u. }\end{array}$ & $\begin{array}{c}\text { Active } \\
\text { Support } \\
\text { Duration } \\
\text { of DFIGs } \\
/ \mathbf{s}\end{array}$ & $\begin{array}{c}\text { Rotor } \\
\text { Speed } \\
\text { Change of } \\
\text { DFIGs } \\
\text { /p.u. }\end{array}$ \\
\hline 10.0 & 0.300 & 0.0162 & 6.59 & 0.0028 \\
\hline 15.0 & 0.368 & 0.0178 & 6.51 & 0.0031 \\
\hline 25.0 & 0.500 & 0.0181 & 6.47 & 0.0032 \\
\hline 35.0 & 0.601 & 0.0183 & 6.35 & 0.0034 \\
\hline 50.0 & 0.905 & 0.0185 & 6.22 & 0.0035 \\
\hline
\end{tabular}

According to the analysis of Fig.10 and Table 1, the short-term additional active output of DFIGs increases, while the duration of active support decreases with the increase of the wind power penetration $\alpha_{\mathrm{w}}$. Therefore, the short-term additional energy of DFIGs is limited and equal under the same operating conditions. In addition, the power drop and the secondary frequency drop of the system increase gradually in the speed recovery stage. And the frequency recovery duration increases gradually, which limits the further improvement of the wind power penetration of the system.

\section{Conclusion}

(1) A variable inertia coordination frequency regulation strategy for wind and thermal joint system with different wind power penetration ratios was established. This strategy considered the difference of the frequency regulation capability and of the frequency regulation effect between DFIGs and thermal power units. Through the study of the equivalent inertia time constant of DFIGs and the stability analysis of the incremental model, the reasonable adjusting range of variable inertia frequency regulation parameters of DFIGs was given.

(2) Under the same wind power penetration conditions, the lowest frequency of the system was raised and the frequency response performance of the system was better when wind farms participated in frequency regulation. At the same time, the auxiliary inertia supplied by DFIGs, which damped the system frequency deterioration and ensured the stability of the whole control system.

(3) The simulation results under different wind power penetration ratios showed that wind farms could reliably assist frequency regulation under various operating conditions and the proposed frequency regulation strategy was robust. Meanwhile, it should be noted that the increase of the wind power penetration ratio made the secondary frequency drop more serious, which was unavoidable. And the phenomenon couldn't be ignored when the wind power penetration exceeded $30 \%$.

\section{Appendix}

Table A The simulation parameter of the incremental system

\begin{tabular}{|c|c|c|}
\hline \multicolumn{2}{|c|}{ Parameter } & Value \\
\hline \multirow{2}{*}{$\begin{array}{l}\text { Frequency } \\
\text { regulation }\end{array}$} & $K_{\mathrm{m}}$ & 0.7 \\
\cline { 2 - 3 } & $\delta$ & 0.05 \\
\hline
\end{tabular}

\begin{tabular}{|c|c|c|}
\hline parameters of the & & \\
thermal power unit & $K_{\mathrm{P}}$ & 1 \\
\cline { 2 - 3 } & $T_{\mathrm{I}}$ & $4 \mathrm{~s}$ \\
\cline { 2 - 3 } & $T_{\mathrm{S}}$ & $0.1 \mathrm{~s}$ \\
\cline { 2 - 3 } & $T_{\mathrm{CH}}$ & $0.25 \mathrm{~s}$ \\
\cline { 2 - 3 } & $T_{\mathrm{RH}}$ & $9 \mathrm{~s}$ \\
\cline { 2 - 3 } & $T_{\mathrm{P}}$ & $0.3 \mathrm{~s}$ \\
\cline { 2 - 3 } & $H_{\mathrm{eq}}$ & $5 \mathrm{~s}$ \\
\cline { 2 - 3 } & $\alpha$ & 0.33 \\
\cline { 2 - 3 } & $D$ & 2 \\
\cline { 2 - 3 } $\begin{array}{c}\text { Frequency } \\
\text { regulation } \\
\text { parameters of the } \\
\text { DFIG }\end{array}$ & $K_{\mathrm{w}}$ & 0.3 \\
\cline { 2 - 3 } & $T_{\mathrm{f}}$ & $5 \mathrm{~s}$ \\
\cline { 2 - 3 } & $K_{\mathrm{f}}$ & 3 \\
\cline { 2 - 3 } & $K_{\mathrm{pf}}$ & $1.0($ variable) \\
\cline { 2 - 3 } & $K_{\mathrm{df}}$ & $1.0($ variable) \\
\cline { 2 - 3 } & $K_{\mathrm{pfp}}$ & variable \\
\cline { 2 - 3 } & $K_{\mathrm{dfp}}$ & variable \\
\cline { 2 - 3 } & $V_{\mathrm{w}}$ & $9 \mathrm{~m} / \mathrm{s}$ \\
\hline
\end{tabular}

\section{References}

1. N. Nguyen, J. Mitra. IEEE Transactions on Sustainable Energy. 7, 354 (2016)

2. B. Andrea, D. Federico, I. Marco, P. Renato. Energies. 10, 102 (2017)

3. X. Tang, M. Yin, C. Shen, Y. Xu, Y. Zhao, Y. Zou. IEEE Transactions on Sustainable Energy. 10, 822 (2019)

4. IEEE Standard for Interconnection and Interoperability of Distributed Energy Resources with Associated Electric Power Systems Interfaces (IEEE Std, 2018)

5. G. Delille, B. Francois, G. Malarange. IEEE Transactions on Sustainable Energy. 3, 931 (2012)

6. F. Hafiz, A. Abdennour. Renewable Energy. 80, 629 (2015)

7. M. Altin, A.D. Hansen, T.K. Barlas, K. Das, J.N. Sakamuri. IEEE Transactions on Sustainable Energy. 9, 1732 (2018)

8. J. Morren, S.W.H.D. Haan, W.L. Kling, J. A. Ferreira. IEEE Transactions on Power Systems. 21, 433 (2006)

9. S. Wang, K. Tomsovic. IEEE Transactions on Power Systems. 33, 6579 (2018)

10. D. Gautam, L. Goel, R. Ayyanar. IEEE Transactions on Power Systems. 26, 214 (2011)

11. Z.A. Obaid, L.M. Cipcigan, L. Abrahim, M.T. Muhssin. Journal of Modern Power Systems and Clean Energy. 7, 9 (2019)

12. S.I. Abouzeid, Y. Guo, H. Zhang. Journal of Renewable and Sustainable Energy. 11, 1 (2019)

13. S. Negri, E. Tironi, D.S. Danna. IEEE Transactions on Industry Applications. 55, 2401 (2019)

14. J. Li, B. Wen, H. Wang. IEEE Access. 7, 39509 (2019)

15. C. Abbey, G. Joos. IEEE Transactions on Industry Applications. 43, 769 (2007)

16. Z. Wu, W. Gao, H. Zhang, S. Yan, X. Wang. IEEE Transactions on Sustainable Energy. 8, 1330 (2017) 
17. A. bonfiglio, M. invernizzi, A. labella, R. Procopio. IEEE Transactions on Power Systems. 34, 754 (2019)

18. J. Zou, M. Pipattanasomporn, S. Rahman. IEEE Transactions on Sustainable Energy. 7, 1583 (2016)

19. P.K. Keung, P. Li, H. Banakar, B.T. Ooi. IEEE Transactions on Power Systems. 24, 279 (2009)

20. S.M. Muyeen, A. Al-Durra. IEEE Systems Journal. 7, 817 (2013) 